\title{
Peruanos en Córdoba: migraciones, talleres textiles y prácticas comunitarias
}

\author{
Peruvians in Cordoba: migration, garment workshops and \\ community practices \\ María José Magliano / mariajosemagliano@gmail.com \\ http://orcid.org/0000-0002-3028-5129 \\ María Victoria Perissinotti / vperissinotti@gmail.com \\ http://orcid.org/0000-0002-0527-4444 \\ Denise Zenklusen / denisezenklusen@gmail.com \\ http://orcid.org/0000-0001-6620-5226 \\ Consejo Nacional de Investigaciones Cientificas y Técnicas (CONICET) \\ Universidad Nacional de Córdoba (UNC), Argentina
}

\begin{abstract}
Based on the findings of an ethnographic research, this paper proposes to analyze the labor trajectories in "informal" garment workshops of Peruvian migrants who live in territories of urban relegation of Cordoba (Argentina), regarding their strategies to access the city and family reproduction. Moreover, it explores the community practices, as resources of local organization intended for self-management, mobilized in neighborhoods in connection with the development of garment workshops. This study shows how some processes that exceed migration itself -such as presence/absence (selective) of the State in urban spaces, informal labor networks and difficulties to access housing- are related to mobility and labor insertions, making it possible and stimulating the existence and proliferation of informal garment workshops in specific places of the city.
\end{abstract}

Key words: urban space, garment workshops, Peruvian migrants, communitarian practices, autonomy of migration.

Resumen: A partir de los resultados de un trabajo de campo etnográfico, este artículo se propone indagar en las trayectorias laborales en talleres textiles "informales" de migrantes peruanos que viven en territorios de relegación urbana de Córdoba (Argentina), en el marco de las estrategias de acceso a la ciudad y de reproducción familiar. Asimismo, reflexiona sobre las prácticas comunitarias, en tanto recursos de organización barrial tendientes a la autogestión, que se despliegan en esos territorios en relación con el desarrollo de los talleres textiles. Este estudio muestra cómo ciertos procesos que exceden la migración en sí misma - como la presencia/ausencia del Estado, las redes de trabajo informal y la dificultad para el acceso a la vivienda - se relacionan con las movilidades e inserciones laborales de la población migrante de forma tal que posibilitan y estimulan la existencia y proliferación de talleres textiles informales en zonas concretas de la ciudad.

Palabras clave: espacio urbano, taller textil, migrantes peruanos, prácticas comunitarias, autonomía de las migraciones. 


\section{Introducción}

A partir de los resultados de un trabajo de campo etnográfico, este artículo se propone indagar en las formas de habitar los espacios urbanos -concebidos como una construcción histórica y política (Wacquant, 2007: 21)-, a partir de analizar el modo en que ciertas trayectorias laborales "migrantes", como el trabajo textil, se articulan con los modos de apropiación de esos espacios, en el marco de la obtención de las estrategias de subsistencia y reproducción familiar.

En particular, recupera las trayectorias laborales de migrantes peruanos, varones y mujeres, que llegaron en las últimas décadas a la ciudad de Córdoba (Argentina) y viven en territorios de relegación urbana. Asimismo, reflexiona sobre los lazos de solidaridad y las prácticas comunitarias que los migrantes despliegan en esos territorios en relación con el desarrollo de los talleres textiles. En los territorios de relegación urbana, esos lazos y prácticas funcionan como un recurso de organización barrial tendiente a la autogestión.

En el caso de los migrantes peruanos, las prácticas comunitarias no provienen de una tradición indígena - como puede suceder en los casos de la migración boliviana reconstruidos en los estudios de Bastia (2007), Colectivo Simbiosis/Colectivo Situaciones (2011) y Gago (2014)-, sino de las estrategias y posibilidades que se activan en los recorridos como migrantes en los lugares de destino. Recorridos que van desde las pensiones a las que arriban, compartidas con otros compatriotas; pasando por las redes que se despliegan en los procesos de llegada tanto a las ciudades de destino como a ciertos espacios dentro de la misma ciudad; hasta el propio desarrollo y mantenimiento del taller textil, el cual se nutre de un "saber-hacer" configurado a partir de la centralidad que cobra la costura como inserción laboral para esta población en la ciudad de Córdoba.

En torno a ello, reconstruiremos las especificidades de Sabattini, un barrio de relegación urbana ubicado en la periferia este de la ciudad de Córdoba y situado sobre terrenos fiscales pertenecientes al Estado nacional. Este espacio se consolida entre los años 2009 y 2010 cuando un grupo de personas, principalmente de origen peruano, comienzan a tomar ${ }^{1}$ los terrenos, ya que no podían continuar costeando los alquileres en otros lugares de la ciudad. La constitución de Sabattini respondería a la categoría de "villa" utilizada en Argentina para referirse al conjunto de predios sobre el que se hizo una sucesión de tomas de tierras, ventas y re-ventas de modo paralegal y sin servicios básicos (Gago, 2014).

1 La letra cursiva será utilizada para destacar categorías nativas. 
Si bien Sabattini cumple con estos "requisitos", en este trabajo nos referimos a dicho espacio como "barrio", pues es así como lo denominan los propios vecinos. En sus relatos surge reiteradamente la idea del esfuerzo realizado por construir un espacio similar a un "barrio" no una "villa", con las implicancias simbólicas que esto posee en los modos de habitar la ciudad (Magliano et al., 2014).

Justamente, es en estos espacios donde los talleres textiles "informales" y "clandestinos" 2 se asientan, proliferan y funcionan sin sobresaltos, en pos de aprovechar los recursos comunitarios, de protección y de fuerza de trabajo allí presentes (Gago, 2014: 232). Ahora bien, el argumento propuesto en este trabajo va más allá de esta premisa: para nosotras la posesión del taller textil "informal" y "clandestino" emerge como condición de posibilidad a partir de la obtención de un lugar para vivir, lo cual sucede a través de un proceso de apropiación del espacio en el contexto de las luchas por el acceso a la ciudad.

En este sentido, las fronteras al interior de las ciudades funcionan no sólo como factor de exclusión, sino también como campo de posibilidades en torno a la búsqueda de estrategias de subsistencia que cuestionan la regulación y control estatal. La misma llegada a Sabattini -en tanto espacio apropiado de manera "ilegal" de acuerdo con los parámetros establecidos por el Estado- puede leerse como resultado de mecanismos de disputa del orden estatal, en el marco de prácticas de "reivindicación del derecho a la ciudad" (Sassen, 2010: 396).

Las reflexiones que guiarán este trabajo se nutren fundamentalmente de dos corrientes teóricas. En primer lugar, retoma las propuestas del enfoque de la autonomía de las migraciones, el cual parte de considerar a la migración como una fuerza creativa y contestataria dentro de las estructuras sociales, culturales y económicas (Papadopoulos et al., 2008).

Desde esta perspectiva, la explotación que enfrentan los migrantes siempre deja margen para la acción que éstos despliegan, como "su condición de posibilidad y la base material de su potencial impugnación" (Mezzadra, 2012: 163). Es decir, de acuerdo con la autonomía de las migraciones, las luchas migrantes refieren a las estrategias cotidianas, a los cuestionamientos

2 Recuperando los planteos de Gago, las categorías “informal” y “clandestino” son concebidas en este trabajo desde su potencial creativo y no sólo desde su connotación negativa. Así, la autora define "informalidad" por su carácter de innovación que busca nuevas formas antes que por su relación con la normativa que define lo legal/ilegal (Gago, 2014: 21) y "clandestino" por una gestión de la mano de obra que excede los parámetros jurídicos y que incluye esferas vitales al interior de un gobierno más amplio del cuerpo y de la subjetividad de quien trabaja (Gago, 2014: 141). 
y a las resistencias a través de las cuales los migrantes expresan su presencia "conflictiva" en los países de destino (De Genova et al., 2015).

En el caso que aquí analizamos, los mecanismos de explotación que enfrentan los migrantes a partir de su posición de desventaja social, política, económica y cultural en las ciudades de destino activan formas de lucha y resistencia. Precisamente, es en estos espacios donde el poder se hace concreto y puede ser desafiado, y donde los oprimidos (en este caso un amplio conjunto de los y las migrantes) son parte de la "infraestructura social para el poder" (Sassen, 2015: 21, énfasis en el original).

En segundo lugar, este estudio recupera los aportes de la sociología urbana para pensar las implicancias de los actuales procesos de desigualdad, marginalidad y resistencia, reconociendo que desde comienzos de este nuevo siglo "la ciudad resurge como espacio estratégico para entender tendencias críticas en la reconfiguración del orden social” (Sassen, 2007: 129).

Aquellos procesos no están tejidos en todos lados con las mismas fibras; por el contrario, los mecanismos genéricos que los producen y las formas específicas que revisten se vuelven inteligibles cuando se los ubica en la matriz histórica de relaciones entre las clases, el Estado y el espacio (Wacquant, 2007). En tal sentido, con el objetivo de contribuir al campo de discusión y estudio sobre las ciudades, el trabajo y las migraciones en las sociedades contemporáneas, este artículo se inspira en las lecturas de Das y Poole (2008), De Genova et al. (2015), Gago (2014), Mezzadra (2012), Sassen (2007, 2010 y 2015) y Wacquant (2007).

El siguiente estudio se organizará en torno a tres apartados. En el primero describiremos la estrategia metodológica seleccionada para el desarrollo de esta investigación. En el segundo, nos detendremos en las formas que adquiere la informalidad para pensar las estrategias que despliegan los migrantes para habitar y subsistir en el espacio urbano. En pos de ello, examinaremos las articulaciones entre los modos de apropiación de Sabattini en la búsqueda de un lugar para vivir y la consolidación de ciertos trabajos, como la costura.

En diálogo con estos aspectos, analizaremos las especificidades de este sector económico en las últimas décadas, en el marco de reconocer las particularidades de los procesos de segmentación del mercado de trabajo a partir de la crisis de legitimidad neoliberal del año 2001 en Argentina. Es decir, cuando aparecen y se reproducen nuevas formas de informalidad bajo un escenario de creciente preeminencia de la dimensión territorial.

En el tercer apartado indagaremos en los lazos de solidaridad y prácticas comunitarias presentes en la conformación y funcionamiento de los talleres textiles en barrios de relegación urbana; así como también sobre las tensiones y 
disputas que emergen en términos de relaciones de poder y desigualdades entre los propios migrantes. Estos lazos y prácticas se vinculan a maneras singulares de apropiación y negociación del espacio urbano y, al mismo tiempo, son fundamentales "para la producción y sostenimiento de formas laborales de intensa explotación” (Gago, 2014: 47).

A pesar de que la relación entre trabajo textil y migraciones se ha transformado en los últimos años en un tema de creciente interés para el ámbito académico en Argentina, este artículo asume el desafío de abordar una arista que ha recibido escasa atención: la articulación entre formas de apropiación del espacio urbano, informalidad de la vida cotidiana y desarrollo de determinadas inserciones laborales por parte de los y las migrantes, peruanos en nuestro caso, que llegaron en el transcurso de la última década al país.

En ese contexto, pretendemos ir más allá de la descripción de las lógicas de explotación que efectivamente sostienen al taller textil, para poder dar cuenta de todo aquello que está detrás de su funcionamiento y que expresa no sólo precariedad sino también creatividad, solidaridad y resistencia. Como sugiere Sassen (2007), es importante recuperar las categorías de lugar y de proceso de producción para observar la multiplicidad de economías - muchas de las cuales son de baja calificación y mal remuneradas- y de culturas del trabajo que conforman el sistema económico global contemporáneo.

\section{La etnografía como estrategia metodológica}

En el caso de Sabattini, el trabajo textil forma parte de microemprendimientos familiares bajo dos modalidades principales: el trabajo como costureros empleados en talleres que se ubican "fuera" del barrio (en general, en la zona céntrica de la ciudad) y el trabajo en la propia casa a partir de la posesión de un taller que puede ocupar no sólo a los miembros de la familia sino a otros vecinos, también peruanos. En ambas modalidades, los migrantes se dedican a la confección de prendas a partir de un modelo que, quien hace de intermediario con el dueño de la marca, les entrega. Estos intermediarios pueden ser tanto de origen argentino como migrante, con trayectorias más "exitosas" dentro de la actividad económica. En general, las partes de las prendas -cortes en términos nativos- llegan ya cortadas, siendo el trabajo principal el armado final de la pieza.

Debido a su informalidad -y en algunos casos, su ilegalidad-, "entrar" a estos talleres no resulta una tarea sencilla. De hecho, no fue sino hasta varios meses después de comenzado nuestro trabajo de campo y luego de haber compartido otros espacios de la cotidianidad de los vecinos, que pudimos "ingresar" a los talleres textiles que conocimos. 
En este sentido, entendemos que la realización de esta investigación no hubiera sido posible sin el intenso trabajo de campo etnográfico llevado a cabo colectivamente en Sabattini desde agosto de 2012 hasta fines del año 2015. La centralidad del trabajo de campo etnográfico para analizar temas como los que abordamos en este estudio radica, siguiendo a Das y Poole (2008: 20), en que al ser "una forma de conocimiento que privilegia la experiencia", permite "introducirse en los dominios de lo social que no son de fácil acceso si se siguen los protocolos formales de los que se sirven otras disciplinas".

En términos de Rokwell (2009: 25), entendemos por etnografía a aquellas investigaciones que "parten de la experiencia prolongada del [investigador] en una localidad y de la interacción con quienes la habitan" y que "producen, como resultado de un trabajo analítico, un documento descriptivo [...] en el cual se inscribe la realidad social no documentada y se integra el conocimiento local". En vistas a construir este tipo de conocimiento, durante el trabajo de campo nos valimos principalmente de dos técnicas de recolección de datos: la observación participante y las entrevistas en profundidad.

Respecto a la primera, entre mediados de 2013 y fines de 2015, visitamos periódicamente cuatro talleres textiles ubicados en Sabattini, construyendo un total de 40 registros de campo. De los cuatro talleres que visitamos, dos poseían empleados ( 2 y 6 empleados, respectivamente) y los otros dos ocupaban solamente a la pareja. En las distintas visitas, pudimos conversar tanto con los dueños de los talleres como con sus empleados (en caso de tenerlos), quienes son también migrantes peruanos que viven en Sabattini.

Esas conversaciones se dieron tanto en lugar de trabajo como fuera de él, aunque siempre en el espacio del barrio. En todos los casos analizados, los talleres (de diferentes dimensiones en relación con la cantidad y tipo de máquinas y empleados que poseen) son resultado de proyectos familiares. Entre las particularidades del funcionamiento del taller textil, encontramos que la tarea de contactar a los intermediarios que residen "fuera" del barrio recae principalmente en los varones dueños del microemprendimiento. Son ellos quienes también suelen arreglar el precio de la pieza a ser cobrado una vez que el trabajo finalice. Por otra parte, la tarea de llevar y traer los cortes al taller recae de manera indistinta en la pareja propietaria del mismo.

Finalmente, al interior del taller los dueños y empleados, varones y mujeres, trabajan "a la par". Cada uno posee su propio lugar y su máquina en el taller, y eso se mantiene cotidianamente. Los dueños, tanto el varón como la mujer, supervisan el trabajo de todos los empleados y resuelven las dudas que se van generando. Asimismo, se reservan el uso de aquellas máquinas más específicas, 
como la collareta y la remalladora, ${ }^{3}$ en caso de que contaran con ella. Y esto porque no todos los talleres de Sabattini cuentan con la misma infraestructura.

Por otra parte, realizamos tres entrevistas en profundidad, pautadas y grabadas, a mujeres que vivían en el barrio y poseían o trabajaban en talleres textiles. Estas entrevistas se realizaron recién hacia el final de nuestro trabajo de campo, cuando la relación de confianza construida con nuestros interlocutores era suficiente como para ahondar en cuestiones relativas a la organización de los talleres.

Asimismo, las conversaciones informales mantenidas en el marco de nuestras visitas al barrio resultaron de central importancia a la hora de profundizar en los temas que aquí tratamos. En este escenario, hemos podido reconstruir las trayectorias migratorias y laborales de las familias migrantes que allí residen, a la vez que comprender las singularidades de ese espacio en relación no sólo con las propias estrategias de subsistencia y resistencia desplegadas por los y las migrantes, sino también con las articulaciones y diálogos que se establecen con el sistema metropolitano en su conjunto. Es decir, con el desarrollo de ciertos sectores de la economía que, como el caso de la industria textil, se encuentran supeditados a la provisión de una mano de obra barata que se concentra en espacios como Sabattini.

En ese sentido, la etnografía como estrategia metodológica brinda herramientas para reconocer la posición de una zona de relegación urbana en una estructura jerárquica de lugares (medida al mismo tiempo en términos materiales y simbólicos) y la función que cumple en la reproducción de un marco espacial, histórico y económico concreto. Pues, como señala Geertz (1973), el espíritu de la etnografía -y sus principales alcances- reside en analizar procesos sociales en tiempos y espacios acotados, privilegiando la indagación profunda de ese objeto, a partir de reconocer que no estudiamos aldeas, sino que estudiamos en aldeas. El principal reto de este marco metodológico consiste entonces en mostrar cómo los procesos globales pueden ser interrogados desde localizaciones particulares.

\section{La informalidad como forma de subsistencia: migraciones recientes, estrategias de apropiación del espacio y consolidación del taller textil}

Nuestro argumento principal propone que la existencia y proliferación del taller textil "informal" y "clandestino" se articula con la existencia de los barrios de relegación urbana, resultando la espacialidad un factor determinante de

3 La collareta es la máquina que permite unir el cuello de las remeras de escote redondo con pecho y espalda; mientras que la remalladora trabaja con tres o cinco hilos que se cosen en zigzag y recto. 
trayectorias laborales y emprendimientos económicos de los migrantes (en este caso provenientes de Perú) en Argentina. En tal sentido, se torna necesario reconstruir cómo se construye y funciona el taller textil al amparo de cómo se construye el barrio de relegación urbana. Luego, nos interesa identificar algunas de las especificidades de la industria textil en la Argentina contemporánea para poder desentrañar las lógicas presentes en la organización de la costura como trabajo migrante.

Inspiradas en la propuesta de Glick-Schiller y Çaglar (2008), y a través de una mirada microsocial de un barrio de relegación urbana de la ciudad de Córdoba, se trata de dialogar con procesos sociohistóricos más amplios que permitan arrojar luz en torno a las singularidades de Sabattini. Estos procesos sociohistóricos se encuentran determinados por un contexto de creciente informalización del trabajo y de la vida cotidiana en su conjunto que se inicia en los años setenta, se profundiza en el transcurso de la década de 1990 y presenta ciertas especificidades en la última década (Antunes, 2011; Basualdo y Esponda, 2014).

En este panorama, la industria textil, reconvertida a partir de la reactivación del sector luego de la crisis de 2001-2002 en un "trabajo migrante", se reconfigura como el paradigma de la precarización e informalidad laboral (Benencia, 2009; Basualdo et al., 2014; Caggiano, 2014). Estas dinámicas no son ajenas al funcionamiento y reproducción de un orden social y económico global sino que, como señala Sassen (2007: 141), "el funcionamiento cotidiano del complejo de servicios altamente especializados, se sostiene en una gran proporción de empleos manuales y mal remunerados que en gran parte son ocupados por las mujeres y los migrantes", como es el caso de los talleres textiles en Argentina.

Sabattini y sus especificidades permiten iluminar algunas de estas premisas. De acuerdo con el Censo de Viviendas, Hogares, Población y sus Características Migratorias (2014), de la totalidad de las personas que residían allí, unas 700 para el año 2014, el 56\% son peruanas, el 34\% son argentinas y el $10 \%$ restante son oriundas de Bolivia y Paraguay. La población argentina está conformada principalmente por niños/as - muchos/as de los cuales son hijos/ as de padres migrantes- y también por migrantes internos, en especial procedentes de las provincias de Buenos Aires, Misiones, Salta y Santiago del Estero.

Los migrantes peruanos que viven en el barrio se concentran en tres sectores de la economía caracterizados por su informalidad, precariedad e inestabilidad: la construcción, el trabajo textil y el trabajo doméstico remunerado. ${ }^{4}$ Un amplio conjunto de los costureros en Sabattini llegaron a Córdoba

4 La migración peruana hacia Argentina en general y Córdoba en particular es un proceso 
a partir de la existencia de redes sociales que orientaron su inserción laboral a partir de vivir en pensiones donde el mismo tallerista que los había traído (en general también peruano) les alquilaba piezas en la misma vivienda donde funcionaba el taller textil (Magliano, 2016).

Con el tiempo, la imposibilidad de costear el alquiler, las dificultades de vivir en familia en esos espacios - donde se convive con muchas personasy las pretensiones de "progreso" familiar activaron la búsqueda de nuevos lugares dentro de la ciudad, emergiendo especialmente como una alternativa concreta terrenos fiscales que al momento de la toma se encontraban deshabitados. En general, la noticia de que se están ocupando terrenos circula de modo informal entre los vecinos de las pensiones y casas en las que viven los familiares y los amigos (Magliano et al., 2014).

Dadas sus trayectorias laborales (principalmente informales) y su pertenencia de clase, los migrantes sólo pueden aspirar a cierto segmento del mercado de la vivienda que se corresponde con "zonas degradadas", a las cuales se accede a través del mercado informal (Terrones-Ribas, 2005: 204). En el caso de Sabattini, un grupo de argentinos delimitaron algunos terrenos, afirmando que eran propietarios de los mismos. Posteriormente, comenzaron a venderlos a precios que resultaban accesibles comparados con los del mercado formal.

Sin embargo, por tratarse de terrenos fiscales, la venta de los terrenos se mantiene en el plano de lo informal y, por tanto, la propiedad sólo es garantizada por la permanencia de los vecinos allí y no por un título que avale la posesión. En términos legales, la ocupación de terrenos corresponde a una "usurpación", práctica que se encuentra penada por la ley en Argentina (Magliano et al., 2014). ${ }^{5}$

A partir de la relocalización urbana en estos espacios, la posibilidad del taller propio adquiere forma y se vuelve algo factible de concretar. La misma informalidad del barrio alienta y promueve el desarrollo de determinadas actividades productivas que se mantienen también en el plano informal. No nos referimos solamente al taller textil, sino a que la multiplicación de pequeños comercios y lugares de comida en Sabattini expresa las variantes creativas de la informalidad en pos de la subsistencia familiar.

fundamentalmente laboral y urbano que se consolida en la década de 1990. Para profundizar sobre las características más salientes de esta migración en Argentina, véanse los trabajos de Cerrutti (2005), Falcón y Bologna (2013) y Rosas (2010).

5 Estos espacios no poseen servicios públicos formalizados. Hasta el año 2015, Sabattini no contaba con servicio de agua potable, alumbrado público, desagües y gas natural. Asimismo, las viviendas cuentan con electricidad porque están conectadas irregularmente (lo que determina la mala calidad del servicio). En el año 2014, el municipio establece un recorrido especial semanal para la recolección de residuos en el barrio. 
Precisamente, nuestra premisa principal sostiene que la construcción de Sabattini fue la condición de posibilidad para la posterior emergencia del taller textil propio, asentado sobre formas de organización familiar y barrial particulares. La "protección" que les garantiza el barrio facilita la reproducción de ciertos trabajos, informales y con altas dosis de precariedad y explotación laboral. En el taller textil se reproducen determinadas condiciones de trabajo vinculadas con las deficiencias de los espacios en donde se ubican los talleres y, también, a las formas en que se desarrolla la tarea, las cuales se sostienen en extensas jornadas laborales y el trabajo a destajo. ${ }^{6}$

En la gran mayoría de los casos, los migrantes peruanos llegaron a Córdoba para trabajar en talleres textiles de otros compatriotas. Desde el mismo momento en que arriban a la ciudad, la expectativa del progreso está presente, la cual viene de la mano de dejar de ser un empleado para pasar a ser el dueño de un taller. De acuerdo con lo que hemos reconstruido en el trabajo de campo -y al igual que los trayectos de los migrantes bolivianos costureros en Buenos Aires (Colectivo Simbiosis/Colectivo Situaciones, 2011)-, la idea del taller propio aparece como estructurante del proyecto migratorio aun sabiendo que previamente deberán trabajar como empleados.

Esta expectativa, a su vez, se encuentra acompañada por la promesa de dejar de ser inquilinos para convertirse en propietarios de una vivienda, teniendo en cuenta que el espacio del taller es al mismo tiempo parte de la casa. De modo que luego de un tiempo de trabajar como "empleados" y de vivir en piezas alquiladas, la opción del taller propio emerge paralela a la oportunidad de la casa propia, a partir de la ocupación de terrenos fiscales que hasta el momento de la llegada de las familias eran terrenos baldíos.

Una vez que se resuelve la cuestión de la vivienda, la compra de las máquinas de coser -que son de distinto tipo de acuerdo con la tarea a realizar sobre la prenda- marca un quiebre respecto al modo de trabajo; es lo que permite el paso de un costurero empleado a un costurero cuentapropista. Esa compra se hace siguiendo dos estrategias principales: al contado en efectivo y/o en cuotas a partir de acceder a préstamos con los mismos dueños de las máquinas.

El reclamo principal de los migrantes respecto a esta última modalidad es el costo de los intereses que deben asumir cuando las máquinas no se compran al contado - las compras y los préstamos se mantienen también dentro del plano informal-. En Sabattini, la mayoría de los talleres que allí funcionan posee de dos a nueve máquinas aproximadamente.

6 El trabajo a destajo supone un acuerdo previo del precio por cada prenda producida: cuanto más rápido se termina con la elaboración de la prenda, más rápido se cobra el dinero, derivando en extensas jornadas laborales. 
Como planteamos con anterioridad, en aquellos emprendimientos con dos máquinas trabaja sólo la pareja (varón y mujer), mientras que aquellos que cuentan con un mayor número de máquinas incorporan a otros empleados, también peruanos, quienes trabajan para los dueños del taller, que a la vez son los dueños de la vivienda. Asimismo, los talleres ubicados en el barrio producen, fundamentalmente, para marcas locales que se consumen "fuera" del mismo. Es en el "centro", en términos espaciales y simbólicos, donde se consume aquello que se produce y fabrica en los talleres textiles de los barrios de relegación urbana.

Ahora bien, para comprender las lógicas detrás del funcionamiento de los talleres textiles "informales" y "clandestinos" en donde trabaja la población migrante y cuyos propietarios son también migrantes, es preciso reconocer las propias desigualdades presentes en los ámbitos urbanos, las cuales se expresan en formas de vivir y habitar la ciudad marcadas por fuertes asimetrías y restricciones que estimulan la reconfiguración de ciertas actividades económicas que se nutren de esas desigualdades. La costura permite dar cuenta de ello.

En Argentina, el rubro textil sufre un primer cambio significativo en la década de 1970. A partir del segundo quinquenio de esa década, la industria textil -y sus grandes empresas, como Grafa, Sudamtex y Alpargatas, que se desarrollaron bajo el modelo de especialización en la producción masiva de bienes indiferenciados para el consumo básico de una clase trabajadora con ingresos creciente- entra en una profunda crisis de estancamiento (Benencia, 2009: 49).

Esta situación de crisis se profundiza en los años noventa, cuando esta industria fue desmantelada como resultado del ingreso masivo de importaciones favorecido por la convertibilidad peso-dólar. Tras la crisis socioeconómica que atravesó la Argentina en los años 2001-2002, el fin de la paridad cambiaria y la devaluación del peso argentino generaron una revitalización de la industria en el transcurso de esa década, aunque sobre nuevas bases: tercerizando su producción en pequeños talleres, cuya mano de obra está conformada, principalmente, por migrantes de los países de la región sudamericana.

Como sostienen Basualdo y Esponda (2014: 34-35), si bien la tercerización existe desde mucho antes de la crisis mundial capitalista de comienzos de los años setenta, luego de este periodo este proceso pasó de ser una forma de relación laboral subordinada para adquirir un rol protagónico dentro del conjunto de las regulaciones del trabajo. La crisis económica llevó a que un amplio porcentaje de los migrantes limítrofes buscaran reacomodarse en determinados espacios económicos en lugar de retornar a sus países. 
En este marco, "la industria textil, de confecciones y del calzado sirvió como refugio para esta población” (Benencia, 2009: 47)..$^{7}$ Es precisamente el último eslabón de la cadena, el de la confección de prendas, el escenario más propicio para que se den situaciones irregulares por las estrategias empresariales tendientes a tercerizar determinados procesos productivos y no responsabilizarse por lo que sucede en los espacios laborales (Barattini, 2010: 463). De manera que las grandes empresas ya no participan directamente en la producción, sino que subcontratan a empresas más pequeñas, persiguiendo un máximo de flexibilidad de la fuerza de trabajo, en cantidades y acordes con las necesidades productivas (Basualdo et al., 2014; Castles, 2013).

En los barrios de relegación urbana, la informalidad y la irregularidad se nutren de la falta de regulación del Estado en el marco de una presencia/ausencia selectiva. Esa selectividad indica que espacios como Sabattini no se encuentran completamente al margen de las prácticas e injerencias del Estado. Así pues, en este barrio de relegación urbana, el Estado (municipal, provincial y nacional) se hace presente a través de agrupaciones sociales y políticas que actúan de intermediarias frente a demandas de los vecinos.

Ante acontecimientos puntuales, como una tormenta o inundación, la presencia estatal se efectiviza con formas de ayuda directa (chapas, colchones, alimentos). Asimismo, las personas que residen en estos espacios son destinatarios de programas y políticas sociales focalizadas que desde el Estado se implementan, con el propósito de "paliar" su situación de vulnerabilidad. Entre ellas, podemos mencionar la Asignación Universal por Hijo (AUH), ${ }^{8}$

7 En el marco de la diversidad de dinámicas y formas de organización del trabajo textil, en este artículo no abordamos - a la vez que discutimos- la categoría de "trabajo esclavo" como categoría para nombrar esas dinámicas y formas. Esta categoría se ha extendido y legitimado para problematizar política y mediáticamente la existencia del taller textil "clandestino" e "informal" en Argentina, en un contexto más general de debate sobre la trata de personas con fines de explotación sexual y laboral. La relación directa entre taller textil y trabajo esclavo invisibiliza otras formas de organización del trabajo informal que, si bien reproducen lógicas de explotación, expresan estrategias y decisiones que toman los migrantes y sus familias en pos de su subsistencia y del "progreso" familiar.

$8 \mathrm{La} A U H$ es un seguro social que funciona en Argentina desde el año 2009 y otorga a personas desocupadas, que trabajan en empleos informales o que ganan menos del salario mínimo, vital y móvil un beneficio mensual por cada hijo menor de 18 años o hijo discapacitado. 
de alcance nacional; el Boleto Estudiantil Gratuito, ${ }^{9}$ de alcance provincial; y el Vale lo Nuestro, de alcance nacional, pero que gestiona la Municipalidad. ${ }^{10}$

Como bien sostiene De Marinis (2011: 89), casos como el de Sabattini muestran la "economización" de las energías del Estado, lo cual remite a un nuevo formato "adelgazado" de actividad estatal que no implica "retirada" o "desaparición". Esa "economización" y "adelgazamiento" del Estado en Sabattini es lo que permite la existencia y reproducción del taller textil, en el marco de la centralidad que adquiere la dimensión territorial como condición de posibilidad del mismo: ninguno de los microemprendimientos textiles del barrio cumple con las normas legales estipuladas para su apertura y mantenimiento, a la vez que permanecen fuera de los circuitos de control estatal.

La dimensión territorial habilita otras formas de regulación que emanan de las propias necesidades de las poblaciones, con el fin de asegurar su subsistencia política y económica (Das y Poole, 2008: 24). Esto se expresa en un "proceso activo de inclusión del trabajo migrante" (Mezzadra, 2012), no a través de su ilegalización, tal como sucede en contextos como Europa y Estados Unidos, sino a través de su informalización.

La gran mayoría de los migrantes que residen en Sabattini se encuentran en condición de regularidad migratoria. De acuerdo con datos estadísticos, y recuperando la categorización que establece la Ley de Migraciones $\mathrm{N}^{\circ}$ 25.871 vigente desde el año 2004, casi el 70\% de la población migrante que allí residía posee la residencia permanente, el $12,7 \%$, la residencia transitoria y el $8,7 \%$, la residencia precaria (Censo de Viviendas, Hogares, Población y sus Características Migratorias, 2014).

Siguiendo con el razonamiento que propone Mezzadra (2012), esto conlleva un proceso de "inclusión diferencial", en el cual la informalidad laboral y de la vida cotidiana en su conjunto- aparece como una condición producida y como un aspecto clave en los procesos de movilidad. Las formas y modalidades de esa informalidad, en especial en el marco de los lazos de solidaridad y las prácticas comunitarias que emergen tanto en el contexto de conformación de Sabattini como en el armado y sostenimiento del taller textil, serán el objeto principal de indagación del próximo apartado.

9 El Boleto estudiantil gratuito fue implementado en el año 2014 por el gobierno provincial y pueden acceder a este beneficio estudiantes regulares, docentes y personal de apoyo que preste servicios en establecimientos educativos públicos y privados con aporte estatal. Incluye a los niveles inicial, primario, secundario y superior, y a los estudiantes de las universidades públicas de la provincia de Córdoba.

10 El Plan Vale lo Nuestro es un programa de fortalecimiento alimentario que reciben familias de bajos recursos que viven en la ciudad de Córdoba. 


\section{Reciprocidad, vecindad y disputas: sobre las prácticas que favorecen la consolidación del taller textil informal}

En el barrio, los talleres textiles ocupan un lugar en las viviendas familiares. Así pues, a lo largo de los tres años que realizamos trabajo de campo en Sabattini, hemos ido observando la transformación de algunas viviendas en función de la construcción y consolidación de los talleres ubicados en ellas. En general, lo primero que los vecinos construyen es una habitación que les permita, en esta dinámica de informalidad, garantizarse un espacio para habitar y del cual no puedan ser expulsados.

Una vez legitimada su presencia y la posesión de ese lote, comienza la construcción de un espacio común que suele coincidir con la cocina y que, a su vez, es el lugar donde se ubican las primeras máquinas. Se trata de espacios reducidos, con escasa o nula ventilación, casi sin luz natural y con conexiones de electricidad en extremo peligrosas. Los cables, entrelazados y colgando a baja altura, hacen que cuando se camina por allí sea necesario hacerlo con cuidado para no chocar con el cableado que conecta las máquinas y la luces que alumbran el lugar. ${ }^{11}$

A medida que comienzan a conseguir cortes -es decir, que establecen vínculos con personas de afuera del barrio que funcionan como intermediarios con las marcas y les proporcionan mercadería para coser-, las familias que aspiran a construir su propio taller van consiguiendo nuevas máquinas. En esta construcción, espacios de la vivienda van siendo relegados para dar lugar a los talleres. Es decir, el taller va ocupando espacios antes previstos para otros fines, como por ejemplo la cocina o nuevos dormitorios.

Sin embargo, procurando diferenciar lo "privado/doméstico" de lo "laboral", los dueños de los talleres despliegan diversas estrategias para dividir y armonizar los espacios. Así, por ejemplo, telas de diversos tipos se disponen a modo de pared para separar la cocina del taller. Del mismo modo, los escasos muebles que existen se disponen de manera tal que puedan dividir el espacio del taller de las habitaciones familiares.

Este reacomodamiento de las casas en función de los talleres debe entenderse a partir de la intrínseca relación que existe en estos barrios entre vivienda, trabajo y familia. Aquello que ocurre en dicho reacomodamiento es como se produce una reorganización familiar en función de la organización del espacio laboral. Y esto porque, tal como observa Gallinati (2014: 93), existe una "dependencia vital entre los dos ámbitos de vida de los migrantes",

11 Para un análisis de las condiciones de insalubridad vinculadas al modo en que se trabaja en los talleres textiles “informales”, véase Goldberg (2013). 
en tanto "sin el espacio doméstico los talleristas no podrían desarrollar su actividad laboral y sin trabajo no podrían mantener siquiera los gastos de aquellas precarias e insalubres viviendas" (Gallinati, 2014: 93).

Al ubicarse el taller dentro de la vivienda, se convierte en un espacio transitado cotidianamente por niños, tanto de la familia que posee el taller como de algunas costureras madres que allí trabajan. Las extensas jornadas laborales que este tipo de trabajo implica (entre 10 y 12 horas diarias) obliga a que aquellas mujeres que no cuentan con nadie que cuide de sus hijos, deban llevarlos al taller mientras trabajan.

Durante nuestro trabajo de campo hemos podido observar cómo junto con la disposición de las máquinas se encuentran también los cochecitos con los bebés ubicados al lado de sus madres mientras éstas cosen. De este modo, el espacio laboral conjuga las tareas productivas con las tareas reproductivas y de cuidado, aun con las limitaciones que esto implica.

Una vez que los talleres comienzan a consolidarse, y para conseguir nuevos cortes, los talleristas requieren no sólo de nuevas máquinas, sino también de máquinas más específicas para poder realizar otro tipo de tareas. La collareta, la única máquina que hace las terminaciones con la precisión que se requiere, se transforma así en la herramienta más preciada. Pero como también es la más costosa, durante el inicio del taller en general los dueños no pueden afrontar su pago. Al mismo tiempo, para lograr el crecimiento de los talleres, es necesario conseguir personas que estén dispuestas a trabajar en condiciones sumamente precarias y, a la vez, ampliar la red de contactos con personas de afuera que les garantice conseguir más cortes.

Es entonces durante el momento en que el taller comienza a crecer cuando aparecen una serie de acciones -a la vez solidarias y competitivas- que, basadas en nociones de reciprocidad, vinculan a los vecinos en una trama de intercambios a los cuales hemos referido como "prácticas comunitarias". Siguiendo a Gutiérrez-Aguilar (2008), entendemos que se trata de maneras de organización de prácticas que plantean formas de autorregulación y autogobierno. Estas prácticas, concebidas como un "hacer social", se sustentan en una "economía de la reciprocidad" (Gago, 2014: 47) que busca poner a disposición "saberes y recursos que hacen posible desarrollar la vida en un momento marcado por la escasez y la fragilidad de los modos establecidos de reproducción social” (Quiroga-Díaz y Gago, 2014: 11).

Como señalábamos, en Sabattini los talleres textiles "informales" y su crecimiento se encuentran cimentados en estas prácticas. Una de las más usuales remite al intercambio transitorio de máquinas de coser, en función de las necesidades de los distintos talleres dentro del barrio. Así, por ejemplo, 
cuando un vecino que no posee una de estas máquinas - como la collaretapero le resulta imprescindible su uso para poder cumplir con algún trabajo que consiguió, la pide prestada a algún otro vecino que disponga de ella.

En la mayoría de los casos el préstamo se realiza sin problemas, quedando implícito que quien recibió la máquina deberá responder a su tiempo con un favor similar cuando el prestador lo necesite. La circulación de máquinas y favores entre los vecinos que poseen talleres ha posibilitado el sostenimiento y el crecimiento de los mismos.

El pandero constituye otra de las prácticas que observamos reiteradamente en el barrio. Se trata de un sistema informal de préstamos de dinero en efectivo, generado a partir de un grupo estable de personas -en este caso vecinos- que aportan mensualmente un monto fijo de dinero, el cual se distribuye, por turnos, entre ellos. Una vez por mes se sortea quién va a recibir el dinero recaudado en esa oportunidad, de modo que, al finalizar el ciclo del pandero, todos hayan recibido el dinero que les corresponde. Lo importante de este sistema es que permite a los vecinos acceder, de una sola vez, a una suma de dinero importante que no podrían conseguir individualmente.

Como nos comentaba una vecina, el beneficio del pandero es que el dinero llega "todo junto". Según nos explicaba, "si tú guardas la plata, te la vas picoteando. En cambio, si se la das así, no la gastas y entonces después te viene toda junta. Es un esfuerzo, pero cuando te llega es muy bueno" (Eliana, migrante peruana, 27/02/2014).

Este sistema de préstamos, que hunde sus raíces en la informalidad y se nutre de las relaciones comunitarias para su expansión, resulta fundamental para la consolidación de los talleres textiles en tanto la suma de dinero que de allí reciben los talleristas les facilita la compra de aquellos bienes más costosos que resultan imprescindibles para la consolidación del microemprendimiento textil. ${ }^{12}$ De modo que el sistema del pandero puede pensarse como una práctica comunitaria en tanto se sustenta en relaciones de confianza y vecindad que necesariamente obligan a la reciprocidad.

Por otro lado, señalábamos también la importancia de conseguir costureros a medida que el taller se va ampliando. En tanto dos personas no son suficientes para responder con la rapidez que el negocio demanda, quienes aspiran a consolidar sus talleres se ven obligados a "contratar" a nuevas personas. En todos los casos que conocimos, quienes cubren esta demanda son vecinos, también de origen peruano, que en ese momento se encuentran desempleados.

12 Para quienes no tienen talleres, pero también participan de este sistema, los autos usados, las motocicletas y los electrodomésticos constituyen algunos de los bienes más deseados y por los cuales ingresan al pandero. 
Así pues, se establece una continua circulación de personas que van ocupando estos trabajos a medida que van quedando disponibles. Y también, en algunas ocasiones, los talleres hacen espacio a vecinos de su confianza que realmente necesitan un ingreso. Sin embargo, dado que los márgenes de ganancia son reducidos, para poder hacer una diferencia hay también una fuerte dosis de explotación en estas relaciones. En una conversación que mantuvimos con una vecina, nos comentaba que le habían ofrecido trabajar en un taller del barrio, pero que, si bien necesitaba el dinero, no estaba convencida de aceptarlo debido a que el pago era muy malo. Nos explicaba entonces:

Me ofrecieron para que cosiera los bolsillos de unos pantalones. Pero me pagan dos pesos por cada uno que cosiera. Y yo estuve calculando, y cada bolsillo me lleva cinco minutos. Entonces para poder juntar algo como un sueldo, tenía que estar todo el día cosiendo. ¡Sabés todo el tiempo que tenía que estar para poder juntar algo de dinero! (Liliana, migrante peruana, 09/10/2014).

En un contexto de precariedad y escasez, el horizonte del trabajo se reorganiza en función y a partir de lazos de vecindad que habilitan y facilitan el desarrollo de dinámicas laborales que se alimentan de esta misma precariedad.

Por último, mencionábamos también la importancia de conseguir y extender la red de contactos hacia aquellas personas que ofrecen cortes en vistas a ampliar la productividad y, por ende, los talleres. Para conseguir dichos contactos es fundamental que alguien conocido presente y recomiende al dueño del taller con estas personas de afuera. Son los mismos vecinos quienes "se pasan" y comparten esos contactos, como un modo de garantizar la confianza de quienes les dan cortes.

Si entendemos a las prácticas comunitarias como acciones de solidaridad que, en escenarios de escasez, buscan incrementar las posibilidades de subsistencia, podría pensarse que esta red de intercambio de contactos, recomendaciones y confianza funciona como tal. No obstante, una vez más, el horizonte de la comunidad contiene también elementos de disputa.

Los talleristas en sus relatos expresan que, ante el ofrecimiento de cortes para trabajar, es fundamental aceptarlos rápidamente y confirmar que van a ser capaces de hacerlo en poco tiempo. "Después uno se las arregla para cumplir - nos comentaba el dueño de un taller-, pero si no le dices que lo tienes rápido, ya se van buscando a otros que lo hagan" (Fermín, migrante peruano, 10/12/2014).

Esos "otros" suelen ser los propios vecinos de Sabattini, por lo que se pueden generar ciertas competencias en relación a quién se queda con los cortes. Aparecen entonces diversas estrategias -como presentar un taller más ordenado y limpio o contratar más personas para terminar en menor tiempo- 
en orden a "ganar" los cortes ofrecidos. Nuevamente, la ambigüedad y ambivalencia de lo comunitario atraviesan las prácticas de los vecinos, en este caso de los talleristas.

$\mathrm{Al}$ mismo tiempo que los lazos de vecindad hacen circular contactos, fuerza de trabajo e incluso los propios bienes que conforman un taller, pueden a la vez tensionar los vínculos a partir de la competencia entre los mismos vecinos en pos de obtener los cortes con el propósito de tornar a los talleres operativos y más "productivos".

En general, quienes trabajan teóricamente con la noción de prácticas comunitarias vinculan este tipo de acciones a formas sociales y lógicas políticas asociadas al mundo de lo indígena. Por el contrario, al rescatar esta noción en esta investigación nos interesa más bien iluminar la "capacidad práctica de las diversas comunidades humanas para cooperar entre sí” de la que habla Gutiérrez-Aguilar (2008: 35).

En este sentido, entendemos -y buscamos mostrar- que en el caso de los talleres textiles que analizamos, las prácticas comunitarias que los sustentan y propician -así como aquellas disputas que los tensionan- tienen un arraigo territorial ligado al espacio del barrio y a su proceso de conformación.

Así, a diferencia de lo observado en los talleres textiles bolivianos en distintos barrios del Área Metropolitana de Buenos Aires (Bastia, 2007; Gago, 2014), en los talleres de Sabattini el "capital comunitario" movilizado para el sostenimiento y reproducción de la vida diaria no tiene que ver con un conocimiento que "viaje" junto con los migrantes desde su origen hasta su destino. Es decir, no está relacionado con un saber "étnico" o "nacional", sino que se construye en estrecha relación con los procesos migratorios y con las modalidades de acceso y construcción del barrio.

Como hemos observado, el "capital comunitario" de estos talleres se constituye alrededor de los lazos de vecindad; lazos creados en el contexto de destino y consolidados en función de la ocupación y toma de un espacio común en vistas a la obtención de un lugar en dónde vivir. Es en este proceso, y ante la ausencia de políticas de Estado, que el esfuerzo por construir un barrio se realiza comunitariamente. Allí es donde el vecinazgo, sostenido en una fuerte identificación nacional, se va configurando como una de las relaciones que estructuran la vida de estos migrantes. Por este motivo, el surgimiento y la proliferación de los talleres textiles en estos espacios sólo pueden comprenderse en su intrínseca relación con la construcción y consolidación del barrio.

Asimismo, las disputas surgidas alrededor de los talleres encuentran su base en esta relación con el espacio barrial. Fundamentalmente, a través de los recursos que fueron conseguidos en función de una movilización comunitaria 
y que luego son usufructuados, al interior de los talleres, de manera privada. Como muestra Gago (2014: 184), "los talleres clandestinos usufructúan las conquistas comunitarias para su propio beneficio".

Así, del mismo modo que en algunas "villas" de Buenos Aires, en Sabattini los servicios de electricidad conseguidos por las luchas de los vecinos que allí residen funcionan "como infraestructura gratuita" para los dueños de los talleres. La cuestión de la luz emerge en este sentido como una de las preocupaciones centrales y como un tema de constantes conflictos. Quienes poseen talleres textiles suelen ser responsabilizados por los cortes abruptos de energía que se suceden una y otra vez en los momentos de mayor consumo.

Existe también cierta persecución hacia ellos: en más de una ocasión escuchamos cómo se responsabiliza a los talleristas de la falta de luz. En este sentido, conversando con una vecina de Sabattini, nos comentó que en una ocasión casi linchan a un tallerista a raíz de que sospechaban que tenía entre 16 y 22 máquinas en su casa. El hombre, indignado con la acusación, se defendió argumentando que sólo tenía cuatro (Mónica, migrante peruana, 25/ 09/2014). Lo central de esta disputa se relaciona con evaluaciones morales de los vecinos sobre cómo utilizar recursos - escasos- conquistados colectivamente.

Se puede observar así la indisoluble relación entre el taller y el barrio. Y es que, como sosteníamos en un comienzo, nuestro planteo reside justamente en que la proliferación del taller textil "informal" y "clandestino" no puede existir sin el barrio. Dicho de otro modo: gracias a la constitución de espacios urbanos - periféricos, informales y en donde el Estado mantiene una presencia/ausencia selectiva y "economizada" - es que los talleres se "acomodan" y reproducen, sumergidos en un tejido social en donde lo comunitario adquiere a la vez potencia y ambigüedad.

\section{Conclusiones}

En este artículo buscamos reflexionar sobre la dimensión territorial que adquieren las trayectorias laborales en la costura de migrantes peruanos de reciente llegada a la ciudad de Córdoba. En especial, la trama argumentativa de este estudio se organizó en torno a la premisa que la informalidad que distingue a las trayectorias de vida de los migrantes peruanos (y no sólo ellos) en las ciudades argentinas, Córdoba en nuestro caso de análisis, funciona como condición de posibilidad para el desarrollo de determinados nichos laborales, como el rubro textil.

En relación con ello, nos mantuvimos atentas a la advertencia de GlickSchiller y Çaglar (2008: 18), acerca de no reducir las dinámicas migratorias 
contemporáneas a una cuestión "étnica" a partir de ignorar los procesos más amplios que modelan las actividades económicas de los habitantes de las ciudades en general y de los migrantes en particular.

Por el contrario, buscamos poner de manifiesto cómo procesos que exceden la migración en sí misma -como la presencias "adelgazadas" del Estado en determinados espacios y contextos (De Marinis, 2011); las redes de trabajo informal; la dificultad para el acceso a la vivienda- se relacionan con las movilidades e inserciones laborales específicas de forma tal que posibilitan y estimulan la existencia y proliferación de talleres textiles "informales" y "clandestinos" en espacios específicos de la ciudad.

Así pues, intentamos dar cuenta de la emergencia de estos talleres no como una cuestión étnica en sí misma -como si los migrantes por ser migrantes hicieran y trabajaran en talleres-, sino como una conjugación de elementos que facilitan ciertos recorridos laborales en los ámbitos urbanos, tanto en los países de origen como de destino. El taller textil, en tal sentido, es parte de la informalidad que atraviesa la cotidianidad de un amplio conjunto de las familias migrantes en las ciudades argentinas, a la vez que resulta unas de las pocas opciones de inserción laboral a la que acceden en el país. Es justamente esa informalidad la que configura al espacio barrial y a lo comunitario como ejes claves de reproducción de la vida.

Al enfocar la mirada en estos talleres desde una perspectiva no normativa, dimos cuenta de la variedad y complejidad de prácticas y lazos que allí se tejen, mostrando que aún en condiciones de existencia adversas, las personas - en este caso, los migrantes peruanos en un barrio de la ciudad de Córdobadespliegan una serie de estrategias en pos de garantizar su reproducción social, familiar y comunitaria a partir de lazos de "vecindad". Lazos que surgen desde y en virtud de un espacio en particular y común: el barrio. Y esto, como analizamos en el primer apartado, se relaciona estrechamente con el recorrido y la trayectoria de estas personas como migrantes.

Por último, entendemos que la comprensión del funcionamiento de estos talleres no sería acabada si no prestásemos atención a los sistemas económicos globales contemporáneos (Sassen, 2007 y 2015) y, particularmente, al sistema económico de la ciudad en especial. Los fenómenos que aquí analizamos deben problematizarse a la luz de la creciente precariedad e informalidad que caracterizan al mercado laboral del siglo XXI. Así, las prendas producidas a bajo costo - gracias a que se fabrican en un espacio signado por la informalidad y a través de una mano de obra "barata" - son consumidas en el centro de la ciudad y por personas de clases medias y acomodadas. 
Si bien en este artículo no hemos ahondado en profundidad sobre estos temas, sería interesante poder rastrear en futuras investigaciones el recorrido de esas prendas desde su producción hasta su consumo. Esto nos hablaría probablemente de las múltiples y variadas formas en que la ciudad se conecta, se relaciona e interactúa, reproduciendo -pero también contestando y desafiando- las desigualdades.

\section{Referencias}

Antunes, Ricardo (2011), "Os modos de ser da informalidade: rumo a uma nova era da precarização estrutural do trabalho?”, en Servicio Social \& Sociedade, núm. 107, Brasil: Cortez Editora Ltda.

Barattini, Mariana (2010), “Trabajo esclavo y organización: el caso de la Unión de Trabajadores Costureros en Argentina”, en Estudios Demográficos y Urbanos, vol. 25, núm. 2, México: El Colegio de México.

Bastia, Tanja (2007), "From Mining to Garment Workshops: Bolivian Migrants in Buenos Aires", en Journal of Ethnic and Migration Studies, vol. 33, núm. 4, Reino Unido: Taylor \& Francis Group.

Basualdo, Victoria y Esponda, María-Alejandra (2014), "La expansión de la tercerización a nivel global a mediados de los años setenta, sus antecedentes históricos y su alcance actual", en Basualdo, Victoria y Morales, Diego [coords.], La tercerización laboral. Origenes, impacto y claves para su análisis en América Latina, Argentina: Siglo XXI.

Basualdo, Victoria et al. (2014), "La tercerización en América Latina en las últimas décadas. Visiones, debates y aportes", en Basualdo, Victoria y Morales, Diego [coords.], La tercerización laboral. Origenes, impacto y claves para su análisis en América Latina, Argentina: Siglo XXI.

Benencia, Roberto (2009), "El infierno del trabajo esclavo. La contracara de las 'exitosas' economías étnicas”, en Avá, núm. 15, Argentina: Universidad Nacional de Misiones.

Caggiano, Sergio (2014), "Desigualdades entrelazadas, luchas divergentes: migración e industria textil en Argentina", en Revista CIDOB d'Afers Internacionals, núm. 106-107, España: Centre for International Affairs.

Castles, Stephen (2013), "Migración, trabajo y derechos precarios: perspectiva histórica y actual”, en Migración y Desarrollo, vol. 11, núm. 20, México: Red Internacional de Migración y Desarrollo.

Censo de Viviendas, Hogares, Población y sus Características Migratorias (2014), Informe final, Argentina: Programa Multiculturalismo, migraciones y desigualdad en América Latina.

Cerrutti, Marcela (2005), "La migración peruana a la Ciudad de Buenos Aires: su evolución y características", en Población de Buenos Aires, núm. 2, vol. 2, Argentina: Argentina: Dirección General de Estadísticas y Censos.

Colectivo Simbiosis/Colectivo Situaciones (2011), De chuequistas y overlockas: una discusión en torno a los talleres textiles, Argentina: Tinta Limón. 
Das, Vena y Poole, Deborah (2008), "El Estado y sus márgenes. Etnografías comparadas", en Cuadernos de Antropología Social, vol. 27, Argentina: Instituto de Ciencias Antropológicas, Universidad de Buenos Aires.

De Genova, Nicholas et al. [eds.] (2015), "New Keywords: Migration and Borders", en Cultural Studies, vol. 29, núm. 1, Reino Unido: Taylor \& Francis Group.

De Marinis, Pablo (2011), "Derivas de la Comunidad: algunas reflexiones preliminares para una teoría sociológica en (y desde) América Latina”, en SINAIS. Revista Eletrônica Ciências Sociais, núm. 9, vol. 1, Brasil: Universidade Federal do Espírito Santo.

Falcón, María del Carmen y Bologna, Eduardo (2013), "Migrantes antiguos y recientes: una perspectiva comparada de la migración peruana a Córdoba, Argentina”, en Migraciones Internacionales, núm.7, vol. 1, México: El Colegio de la Frontera Norte.

Gago, Verónica (2014), La razón neoliberal. Economias barrocas y pragmática popular, Argentina: Tinta Limón Ediciones.

Gallinati, Carla (2014), Migración, vivienda e integración regional: Un abordaje desde la villa miseria, Tesis para optar por el grado de Doctora en Antropología, Argentina: Facultad de Filosofía y Letras, Universidad de Buenos Aires, mimeo.

Geertz, Clifford (1973), Thick description: Toward an interpretive theory of culture: selected essays, Estados Unidos: Basic Books.

Glick-Schiller, Nina y Çaglar, Ayser (2008), "Migrant Incorporation and City Scale: towards a theory of locality in migration studies", en Willy Brandt Series of Working Papers in International Migration and Ethnic Relations, núm. 2, Suecia: Malmö University.

Goldberg, Alejandro (2013), "Un abordaje comparativo en torno a la incidencia de la tuberculosis en inmigrantes bolivianos de Buenos Aires y San Pablo", en Revista Interdisciplinar de Mobilidade Humana (REMHU), vol. 21, núm. 40, Brasil: Centro Scalabrianiano de Estudos Migratórios.

Gutiérrez-Aguilar, Raquel (2008), Los ritmos del Pachakuti. Movilización y levantamiento popular-indigena en Bolivia, Argentina: Tinta Limón.

Magliano, María-José (2016), "Varones peruanos en Argentina y trayectorias laborales en costura. Masculinidades, roles de género y organización del trabajo en contextos migratorios", en Universitas Humanistica, Colombia, núm. 81, Colombia: Editorial de la Pontificia Universidad Javeriana.

Magliano, María-José et al. (2014), "Estrategias en torno a las formas de apropiación y organización del espacio en un 'barrio de migrantes' de la ciudad de Córdoba, Argentina”, en Revista Estudios Demográficos y Urbanos, vol. 29, núm. 3, México: El Colegio de México.

Mezzadra, Sandro (2012), "Capitalismo, migraciones y luchas sociales. La mirada de la autonomía”, en Nueva Sociedad, núm. 237, Argentina: Fundación Foro Nueva Sociedad.

Papadopoulos, Dimitris et al. (2008), Escape Routes. Control and Subversion in the 21st Century, Inglaterra: Pluto Press.

Quiroga-Díaz, Natalia y Gago, Verónica (2014), "Los comunes en femenino. Cuerpo y poder ante la expropiación de las economías para la vida”, en Economía y Sociedad, vol. 19, núm. 45, Costa Rica: Escuela de Economía de la Universidad Nacional de Costa Rica.

Rockwell, Elsie (2009), La experiencia etnográfica: historia y cultura en los procesos educativos, Argentina: Paidós. 
Rosas, Carolina (2010), Implicaciones mutuas entre el género y la migración. Mujeres y varones peruanos arribados a Buenos Aires entre 1990 y 2003, Buenos Aires: Eudeba.

Terrones-Ribas, Albert (2005), "Política social y exclusión residencial de las mujeres inmigrantes en Madrid y Barcelona”, en Solé, Carlota y Flaquer, Lluis [eds.], El uso de las politicas sociales por las mujeres inmigrantes, España: Instituto de la Mujer.

Sassen, Saskia (2015), Expulsiones. Brutalidad y complejidad en la economía global, Argentina: Katz ediciones.

Sassen, Saskia (2010), Territorio, autoridad y derechos. De los ensamblajes medievales a los ensambles globales, Argentina: Katz ediciones.

Sassen, Saskia (2007), Una sociología de la globalización, Buenos Aires: Katz ediciones.

Wacquant, Loï (2007), Los condenados de la ciudad. Gueto, periferias y Estado, Argentina: Siglo XXI.

María José Magliano. Doctora en Historia por la Universidad Nacional de Córdoba (UNC), Argentina. Investigadora Adjunta del Consejo Nacional de Investigaciones Científicas y Técnicas (CONICET) con lugar de trabajo en el Centro de Investigaciones y Estudios sobre Cultura y Sociedad (CIECSCONICET/UNC). Es Profesora de la Escuela de Historia de la Facultad de Filosofía y Humanidades de la UNC. Posdoctorado en Ciencias Sociales en la Universitá degli Studi di Padova, Italia. En la actualidad coordina el Programa de Investigación "Multiculturalismo, migraciones y desigualdad en América Latina” (CIECS-CONICET/UNC). Líneas de investigación: migraciones, género y trabajo; migraciones e interseccionalidad; migraciones y cuidados; migraciones y derecho a la ciudad. Publicaciones recientes: "Varones peruanos en Argentina y trayectorias laborales en costura. Masculinidades, roles de género y organización del trabajo en contextos migratorios", en Universitas Humanistica, núm. 81, Colombia (2016); "Interseccionalidad y migraciones: potencialidades y desafíos", en Revista Estudos Feministas, vol. 23, núm. 3, Brasil (2015); (en coautoría con A. I. Mallimaci), "Las edades de la migración boliviana en Argentina: Córdoba y Ushuaia como destino", en Revista Si somos americanos. Revista de Estudios Transfronterizos, vol. XV, núm. 1, Chile (2015).

María Victoria Perissinotti. Becaria Doctoral del Consejo Nacional de Investigaciones Científicas y Técnicas (CONICET) con lugar de trabajo en el Centro de Investigaciones y Estudios sobre Cultura y Sociedad (CIECS-CONICET/UNC). Licenciada en Comunicación Social por la Universidad Nacional de Córdoba. Doctoranda en Ciencias Antropológicas por la UNC y Maestranda en Antropología por la misma universidad. Es Profesora del Departamento de Antropología de la Facultad de Filosofía y Humanidades 
de la UNC. En la actualidad es integrante del Programa de Investigación "Multiculturalismo, migraciones y desigualdad en América Latina" (CIECS-CONICET/UNC). Líneas de investigación: participación política de mujeres migrantes, migración y derecho a la ciudad. Publicaciones recientes: "Un lugar donde vivir. Las luchas migrantes por el acceso al espacio urbano en la ciudad de Córdoba (Argentina)", en Revista Interdisciplinar da Mobilidade Humana, núm. 47, Brasilia (2016); (en coautoría con M. J. Magliano y D. Zenklusen), Los nudos ciegos de la desigualdad. Diálogos entre migraciones y cuidado, Buenos Aires: CONICET (2016); (en coautoría con M. J. Magliano y D. Zenklusen), "Estrategias en torno a las formas de apropiación y organización del espacio en un 'barrio de migrantes' de la ciudad de Córdoba”, en Revista Estudios Demográficos y Urbanos, vol. 29, núm. 3 (2014).

Denise Zenklusen. Becaria Doctoral del Consejo Nacional de Investigaciones Científicas y Técnicas (CONICET) con lugar de trabajo en el Centro de Investigaciones y Estudios sobre Cultura y Sociedad (CIECS-CONICET/ UNC). Licenciada en Comunicación Social por la UNC. Doctoranda con mención en Antropología de la Universidad de Buenos Aires y Maestranda en Antropología Social de la UNC. En la actualidad es integrante del Programa de Investigación "Multiculturalismo, migraciones y desigualdad en América Latina” (CIECS-CONICET/UNC). Líneas de investigación: migración, género y juventud; migración, experiencia urbana y desigualdad. Publicaciones recientes: (en coautoría con M. J. Magliano y M. V. Perissinotti), "Las luchas de la migración en contextos laborales: la experiencia de sindicalización de una trabajadora doméstica peruana en Córdoba, Argentina”, en Revista Trabajo y Sociedad, núm. 28, Argentina (2017); "De cómo la familia de Marta organiza el cuidado. Un análisis sobre la relación entre familias migrantes, género y cuidado", en Odisea. Revista de Estudios Migratorios, núm. 3 (2016); (en coautoría con María Victoria Perissinotti), “'No aptos para vivir? Una aproximación etnográfica a las disputas por significar el territorio de un asentamiento de la ciudad de Córdoba, Argentina”, en Etnográfica, vol. 18, núm. 3 (2014).

Recepción: 18 de enero de 2016.

Aprobación: 19 de octubre de 2016. 